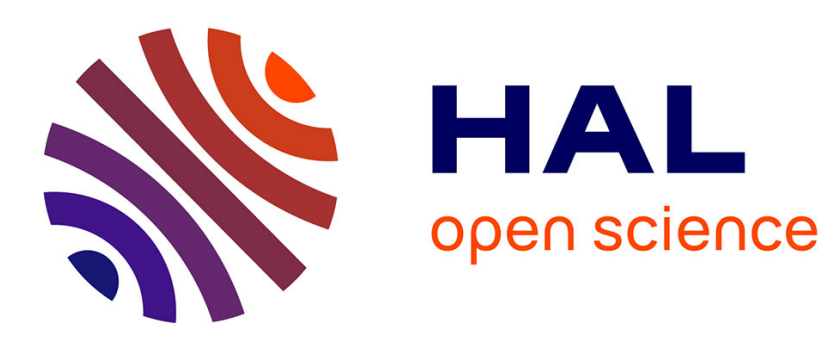

\title{
Photoacoustic laminated characterization of dynamic integrated circuits
}

\author{
J.-W. Fang, S.-Y. Zhang, J.-C. Cheng
}

\section{To cite this version:}

J.-W. Fang, S.-Y. Zhang, J.-C. Cheng. Photoacoustic laminated characterization of dynamic integrated circuits. Journal de Physique IV Proceedings, 1994, 04 (C7), pp.C7-199-C7-202. 10.1051/jp4:1994748 . jpa-00253277

\section{HAL Id: jpa-00253277 https://hal.science/jpa-00253277}

Submitted on 1 Jan 1994

HAL is a multi-disciplinary open access archive for the deposit and dissemination of scientific research documents, whether they are published or not. The documents may come from teaching and research institutions in France or abroad, or from public or private research centers.
L'archive ouverte pluridisciplinaire HAL, est destinée au dépôt et à la diffusion de documents scientifiques de niveau recherche, publiés ou non, émanant des établissements d'enseignement et de recherche français ou étrangers, des laboratoires publics ou privés. 
JOURNAL DE PHYSIQUE IV

Colloque C7, supplément au Journal de Physique II, Volume 4, juillet 1994

\title{
Photoacoustic laminated characterization of dynamic integrated circuits
}

\author{
J.-W. Fang(1), S.-Y. Zhang and J.-C. Cheng \\ Institute of Acoustics and Lab. of Modern Acoustics, Nanjing University, Nanjing 210093, China
}

\begin{abstract}
The layered photoacoustic imaging of dynamic integrated circuits(IC) devices is studied experimentally and theoretically. The experimental method can be used not only to investigate the surface and subsurface structures, but also to analyze the operation modes of the electronic components and the operation principles of IC devices.
\end{abstract}

\section{INTRODUCTION}

Photoacoustic(PA) and photothermal(PT) phenomena in semiconductors have been widely used to study the characterization of semiconductors [1,2], and also the surface and subsurface features of semiconductor materials and devices[2,3]. Meanwhile, the theoretical studies related to PA and PT depth profiling have also attracted remarkable attention[1-4]. In most previous researches, however, the devices are imaged in static states (not in operation), in which some structures having similar electric properties can not be distinguished apparently. In this paper, we present the layered PA imaging of integrated circuits(IC) in dynamic states (in operation). In addition, we consider the influence of the DC electric field on the PA effect in semiconductors, and propose a one-dimensional model to study the effect of the DC electric field on the PA depth profiling. The theoretical results give a good explanation for the experimental phenomena

\section{EXPERIMENT}

An IC device (model T076, D trigger) with logical circuits (a part of the device) shown in Fig.1(a) is used as a sample. The PA imaging of the sample is obtained in the piezoelectric PA microscopy described previously[2]. A static PA magnitude image of the investigated areas of the IC (i.e. the device is in static states) is shown in Fig.1(b). By comparing Fig. 1(b) with (a), it can be illustrated that the marked "A"-"H" areas in Fig. 1(b) are transistors. Some layered PA images of the same areas under the operating voltage are obtained by setting the different phase shifts of the reference signal in the lock-in amplifier, which display the depth profile structures [as shown in Figs.1(c)-(f)]. In the Figs.1(c)-(f), the grey grade is corresponding to the intensity of the positive PA signal, but the white to negative one. The operation states of the sample are that the output of " $A$ " is a low level (logic 0 level) and "B" is a high level (logic 1 level). From the images, we can see that: (1) Some components and areas which are indistinct or even can not be seen in the static PA imaging, such as the resistance " $R$ " and some doped areas of the transistors, can clearly be observed. The images of the transistors operated in the saturation mode (e.g. "A" and "G") are similar to the nearby non-doped regions, but 
some transistors in cut off (e.g. "B", "C" and "H") can be distinguished. (2) Some subsurface features of the sample, such as the depth profilings of the doped areas and aluminum electrodes etc., can be observed obviously. Specially, when the reference phase shift is adjusted to $145^{\circ}$ [Fig.1(f)], some aluminum electrodes are indistinct or even completely disappear. It may reflect that the thickness of all aluminum electrodes in the sample or the structures under the electrodes are not same. In addition, the dark and/or white boundary lines reflect the lateral p- $n$ junctions of the doped areas[5].

\section{THEORY}

A simplified one-dimensional model of the n-type semiconductor sample and the PZT transducer bonded on the back side of the sample is used, in which, a laser beam with modulation frequency $\omega$ illuminates the sample and a uniform electric field is applied to the sample parallel to the surface. Here the influence of the transverse electric field on the transducer is negligible. The thickness of the sample is much larger than the diffusion lengths of photo-generated carriers $(P G C)$ and thermal wave due to the high modulation frequency $(114 \mathrm{kHz})$, and the sample can be considered as a semi-infinite space for the propagation of the plasma and thermal wave.

The PGC diffusion equation and the boundary conditions can be written as

$$
\begin{gathered}
\frac{\partial P(x, t)}{\partial t}=D_{p} \frac{\partial^{2} P(x, t)}{\partial x^{2}}-\frac{P(x, t)}{\tau}+\frac{\beta I_{0}}{2 h v}(1+\cos \omega t) \exp (-\beta x) \\
\left.D_{p} \frac{\partial P(x, t)}{\partial x}\right|_{x=0}=S P(0, t),\left.\quad P(x, t)\right|_{x \rightarrow \infty}=0
\end{gathered}
$$

where $P(x, t)$ is the PGC density, $D_{p}$ is the ambipolar diffusion coefficient, $S$ and $\tau$ are the surface recombination velocity and the lifetime of PGC respectively, $\beta$ is the optical absorption coefficient of the sample, $I_{o}$ and $h v$ are the intensity and photon energy of the incident pump beam respectively.
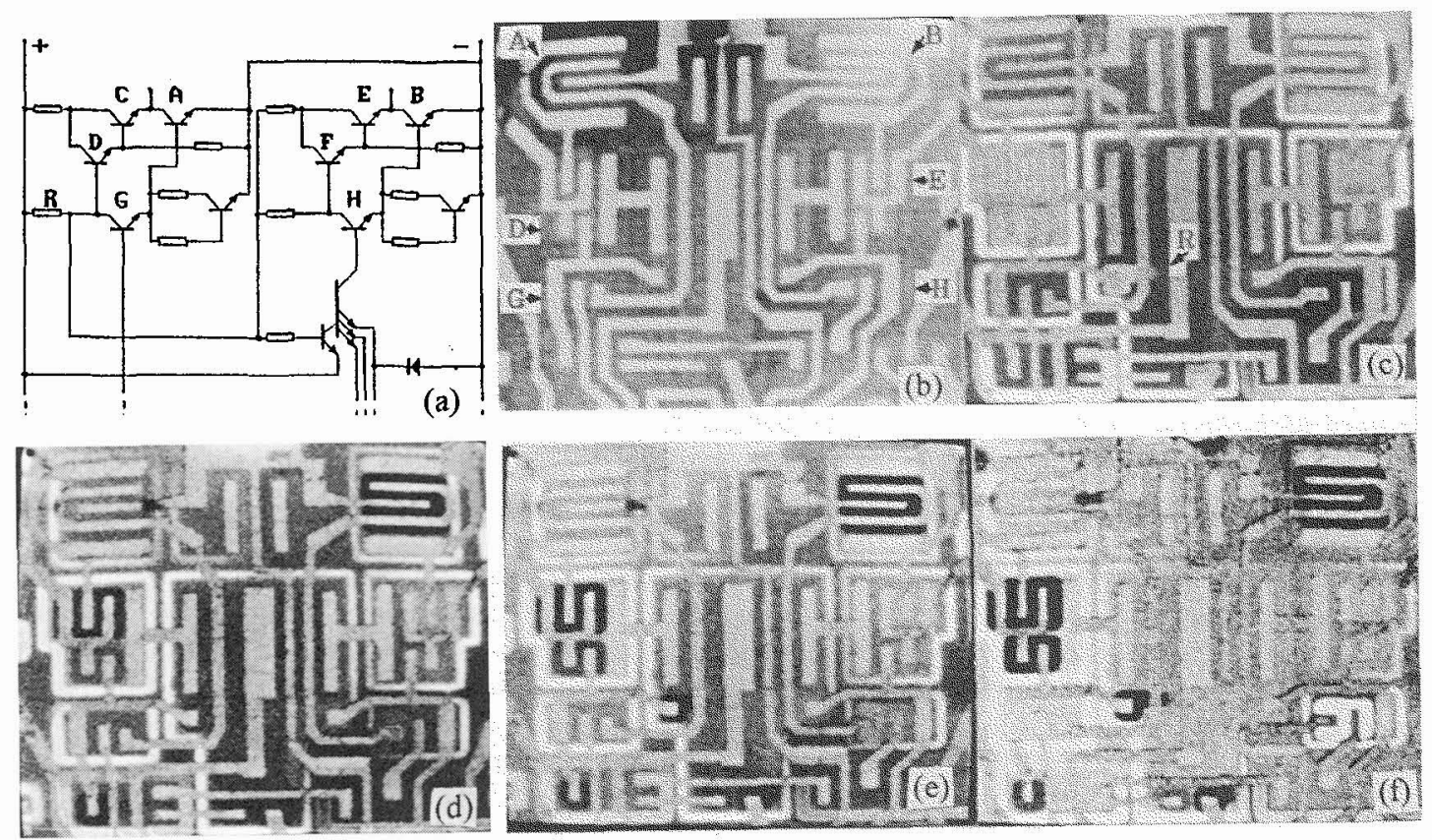

Fig.1 Logical circuit of imaged area of the IC device (a); The static PA magnitude image of the sample(b); Dynamic PA images of the sample at different phase shifts: (c) $65^{\circ},(d) 105^{\circ},(e) 115^{\circ},(f) 145^{\circ}$. 

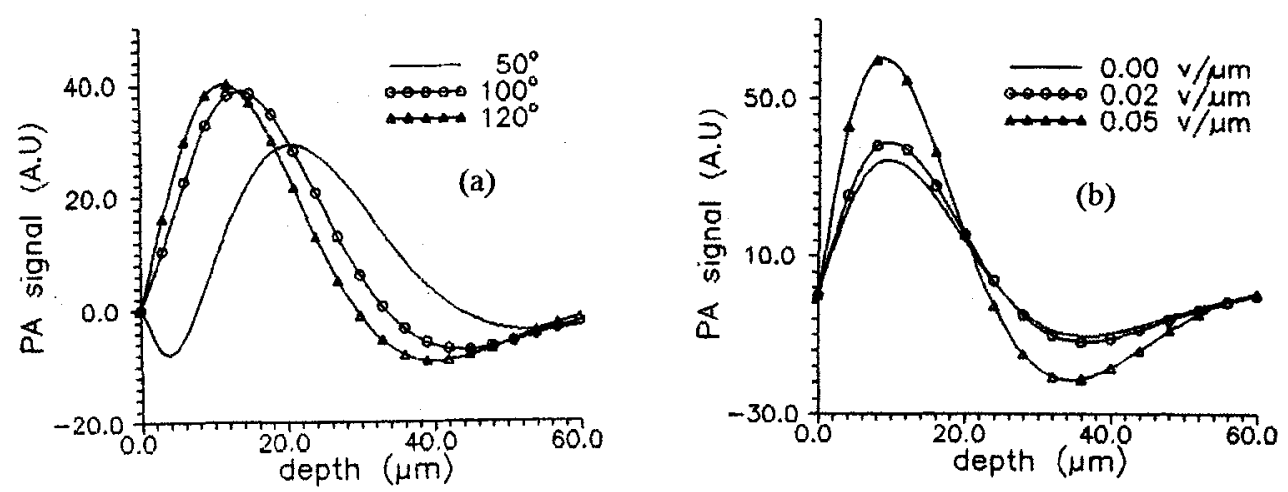

Fig. 2 Contributions of the sources at the different depths to the PA signals, (a) for the different phase shifts as the electric field $0.02 \mathrm{~V} / \mu \mathrm{m}$, and (b) for the different electric fields while the phase shift $140^{\circ}$.

The thermal wave equation and the boundary conditions are as follows

$$
\begin{gathered}
\frac{\partial T(x, t)}{\partial t}=D_{t} \frac{\partial^{2} T(x, t)}{\partial x^{2}}+\frac{E_{g} D_{t}}{K_{t} \tau} P(x, t)+\frac{e E^{2} D_{t}}{K_{t}}\left(\mu_{n}+\mu_{p}\right) P(x, t)+\frac{h v-E_{g}}{2 h v K_{t}} D_{t} \beta I_{0} \exp (-\beta x+j \omega t) \\
\left.K_{t} \frac{\partial T(x, t)}{\partial x}\right|_{x=0}=-S E_{g} P(0, t),\left.\quad T(x, t)\right|_{x \rightarrow \infty}=0
\end{gathered}
$$

where $T(x, t)$ is the temperature variation, $D_{t}$ is the thermal diffusion coefficient, $K_{t}$ is the thermal conductivity, $\mu_{n}$ and $\mu_{p}$ are drift mobilities of electron and hole respectively, $e$ is the electron charge, $E_{g}$ is the energy band gap, and $E$ is the applied DC electric field. Here, the third term on the right-hand side in Eq.(2a) represents the instantaneous Joule thermalization of PGC in the applied electric field. Eqs.(1) and (2) can be solved easily[3].

PA signals in semiconductors are mainly generated by the thermal-elastic expansion and electronicelastic strains[6]. Based on the elastic response and piezoelectricity function, the output voltage of the PZT induced by the source at depth $x^{\prime}$ can be written as[3]

$$
V\left(x^{\prime}, t\right)=\frac{2 K^{2} x^{\prime}(3 \lambda+2 \mu)}{L(\lambda+2 \mu)}\left[\alpha_{t} T\left(x^{\prime}, t\right)+\alpha_{p} P\left(x^{\prime}, t\right)\right] \sum_{n=1}^{\infty} \frac{L n \pi}{(K L)^{2}-(n \pi)^{2}} \int_{i}^{L} \sin \left(\frac{x n \pi}{L}\right) d x
$$

where $\alpha_{t}$ is the thermal expansion coefficient, $\alpha_{p}$ is the electronic-strain constant, $K$ is the acoustic wave vector, $l$ and $L-l$ are the thicknesses of the sample and PZT respectively. The contribution of the acoustic source at $x^{\prime}$ to the output of the lock-in amplifier (i.e. the total PA signal) is

$$
V_{0}\left(x^{\prime}\right)=\left|V\left(x^{\prime}, t\right)\right| \cos \left[\phi\left(x^{\prime}\right)+\phi_{0}-\varphi_{0}\right]
$$

where $\phi\left(x^{\prime}\right)$ is the phase shift of $V_{o}\left(x^{\prime}, t\right), \varphi_{o}$ is the phase shift of the reference signal (i.e. reference phase shift ), and $\phi_{o}$ is the additional phase shift induced by the experiment system. For a certain experiment system, the $\phi_{o}$ is a constant. It can be seen from Eq.(4) that the contribution of the interesting layer in the sample to the imaging signal can be weakened or strengthened by changing the reference phase shift and then the PA depth profiling of the sample is obtained.

\section{DISCUSSION}

\subsection{Influence of the Electric Field on the PA Depth Profiling}

Some numerical calculation results are shown in Fig.2. It is indicated from Fig.2(a) that $V_{o}\left(x^{\prime}\right)$, the imaging signal from the sources at $x^{\prime}$, changes with the reference phase shift. From the curves we can 
see that the investigated depth becomes more shallower as the reference phase shift increases from $50^{\circ}$ to $120^{\circ}$. However, if the reference signal is set in some phase shifts (e.g. $\varphi_{o}<50^{\circ}$ ), the signals caused by the acoustic sources near the sample surface will be negative, and then the images will be white in our experiments since it is the main contribution to total PA signals. Fig.2(b) indicates that $V_{o}\left(x^{\prime}\right)$ changes with the external DC electric fields. The curves can be explained as follows: (1)The PA signals increase with the electric field. If the electric field is strong enough, the imaging region can be shown outstandingly. In our experiments, the resistor " $R$ " can only be seen in the dynamic imaging. Since the external electric field applied to " $R$ " is about $0.05 \mathrm{~V} / \mu \mathrm{m}$ where the two related transistors ("A" and "G") are in the saturation mode. (2)The electric field can affect the phase shift of acoustic source signals due to the Joule thermalization of PGC. Thus we can deduce that, for a certain reference phase shift, the detected depths are different with different electric fields.

\subsection{Layered PA Imaging of Transistor Regions}

The incident pump beam produces the additional influence for the operation mode of the transistor component, and the PA signal is related to the operation mode and electronic environment of the investigated transistor[5]. Then, we can discuss as follows: (1) The internal electric field on the saturated transistor is very small, thus, the PA depth profiling in the component region is similar to the nearby non-doped areas. When the transistor component, originally in the cut off mode, is illuminated by the pump beam, the PGC produces the Joule heat. Meanwhile, the photo-voltage caused by the pump beam may drive the operation mode from the cut off into the "amplification" mode, and then the transistor amplifies the photo-current. While the photo-current passes through the load in the device, the Joule heat is generated. In the layered PA imaging, the transistor can be seen clearly even it is in the cut off mode originally. (2) When the operation mode of the transistor, as " $\mathrm{H}$ ", is driven into "amplification" mode by the pump beam, some related transistors (i.e. "B", " $E$ " and " $F$ " etc.) change their original operation modes[see Fig.(1a)]. The photo-current produced by the pump beam at the " $\mathrm{H}$ " may be continuously amplified by other transistors and the additional acoustic signal is generated. If the pump beam is incident on the transistor " $\mathrm{B}$ ", however, the pump beam will change the operation mode of " $B$ " only. So the phenomena of layered imaging of them are different. For the similar reason, the layered PA imaging of " $D$ " is different with " $E$ " and " $F$ ".

\section{CONCLUSION}

As the IC device is in operation state, the subsurface features in different depths can be strengthened by setting the different phase shifts, which is similar to the condition of the sample in static state. On the other hand, by means of the Joule thermalization of PGC, the electric field influences on the magnitude of the PA signal and the detected depth. Therefore, the layered PA imaging of the dynamic IC device can show the effects of the electric field on the sample and the difference in the operation mode and/or electronic environment between the components, and then the operation principle and some failure factors of the IC device can also be analyzed.

\section{REFERENCES}

[1] A. Rosencwaig, Photoacoustic and Thermal Wave Phenomena in Semiconductors, Edited by A. Mandelis (Elsevier, New York, 1987), pp. 97-135.

[2] S. Y. Zhang \& L. Chen, . ibid, pp. 27-52.

[3] Y. C. Shen \& S. Y. Zhang, IEEE Trans. on UFFC, 39 (1992) 227-231.

[4] J. Opsal \& A. Rosencwaig, Appl. Phys. Lett., 47 (1985) 498-500.

[5] J. W. Fang, Y . C. Shen, Y. S. Lu, J .C. Cheng \& S. Y. Zhang, Acoustical Imaging, Vol. 20, Eds, Y. Wei \& B. Gu, (Plumen, N.Y., 1993), pp.321-327.

[6] G. S. Kino \& R. G. Stearn, Appl. Phys. Lett., 47 (1985) 1048-1050. 